\title{
The evolution of new hospital strains of Staphylococcus aureus
}

\author{
M. PATRICIA JEVONS AND M. T. PARKER \\ From the Cross-infection Reference Laboratory, Colindale, London
}

SYNOPSIS The emergence of new groups of strains of Staph. aureus as important causes of endemic hospital infection in Great Britain has been followed by the phage typing method. Experiments are reported which suggest the possible origin of one of them.

Staphylococcus phage typing is a practical method of characterizing the strains of Staph. aureus frequently isolated from human sources. The basic set of typing phages used for this purpose is chosen so that as many as possible of the important strains pathogenic for man, and particularly those causing endemic sepsis in hospitals, show characteristic patterns of lysis. In the course of time, however, the strains responsible for sepsis in hospitals change, and the new ones may not be typable with the phages in current use. On several occasions since 1950 , the appearance of a new organism has made it necessary to introduce a fresh phage into the basic set of typing phages (Blair and Williams, 1961; Parker, 1962; Report, 1963).

In order to increase the percentage of typable strains, cultures which show no lysis by the basic set of typing phages at their routine test dilution (R.T.D.) are tested with the same phages at 1,000 times this strength (R.T.D. $\times 1,000)$. The reactions given by these strong phage preparations include, as well as those due to phage lysis, areas of general thinning of bacterial growth where a phage filtrate has been applied. These inhibition reactions (Anderson and Williams, 1956) are believed to be due to phage adsorption which leads to bacterial death but not to the production of new phage particles. They are often difficult to distinguish from true phage lysis with secondary overgrowth, and indeed this can only be done with certainty by titration of the phage on the culture. If the reaction is one of inhibition, the thinning of growth will disappear progressively as the strength of the phage is decreased, without the appearance of plaques; if it is a true lytic reaction separate plaques will be seen at an intermediate dilution.

The production of inhibition reactions by a

Received for publication 4 January, 1964. particular phage is a fairly constant property of a staphylococcus, but it is more variable than true lysis, as it is somewhat dependent on the thickness of the bacterial lawn. Thus, though the presence of a series of inhibition reactions with phages of a particular group may be of help in identifying an otherwise untypable staphylococcus, it is undesirable to characterize strains by specific patterns of inhibition.

\section{STRAINS OF TYPE 83A}

In 1958, groups of epidemiologically related infections due to untypable staphylococci, all of which showed a series of inhibition reactions with group III phages, were observed. On the assumption that all the outbreaks were due to 'the same' organism a search was made for a phage which would characterize the new strain by a lytic reaction, and it was found that the phage we now call 83A lysed them all (Fig. 1). This phage had been isolated in the United States several years before by Dr. J. E. Blair and named VA4. Some workers had found it useful in helping to clarify the pattern reactions of certain hospital strains, but organisms lysed only by VA4 had not often been seen.

Phage 83A was first included in the Colindale typing set in 1958, and in that year $6 \%$ of all 'epidemics' were due to type 83A. A retrospective examination of our records suggests that they were probably quite rare before this time. Figure 2 shows that in the years following 1958 there was a steady increase in the proportion of 'epidemics' of hospital sepsis due to type 83A staphylococci (see also Williams and Jevons, 1961). They caused many cases of wound infection, pneumonia, and generalized infection, as well as occasional cases of staphylococcal enterocolitis. Staphylococci of type 83A have 


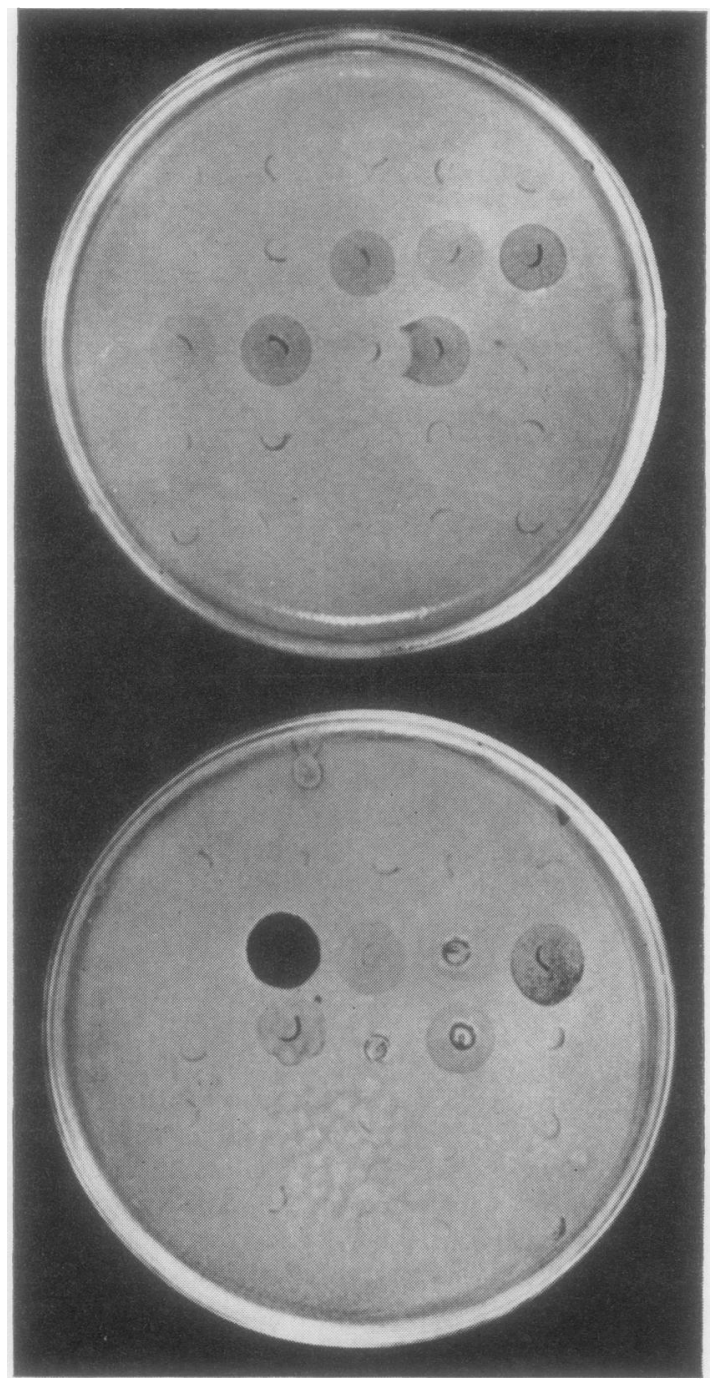

FIG. 1

FIG. 1. A (Above) Staphylococcus aureus type $83 A$. Phage-typing plate $($ R.T.D. $\times 1,000)$ showing confluent lysis by phage $83 \mathrm{~A}$ and inhibition reactions with other group III phages.

$B$ (Below) The new untypable strain of Staphylococcus aureus. Phage-typing plate $($ R.T.D. $\times 1,000)$ showing a pattern of inhibition reactions similar to that of the $83 \mathrm{~A}$ strains.

FIG. 2. Percentage of epidemics of hospital sepsis due to (a) Staph. aureus strains lysed only by phage $83 \mathrm{~A}$ and (b) untypable strains with inhibition reactions similar to those of the 83A staphylococci. An epidemic was defined as a group of three or more related cases of sepsis in one hospital.
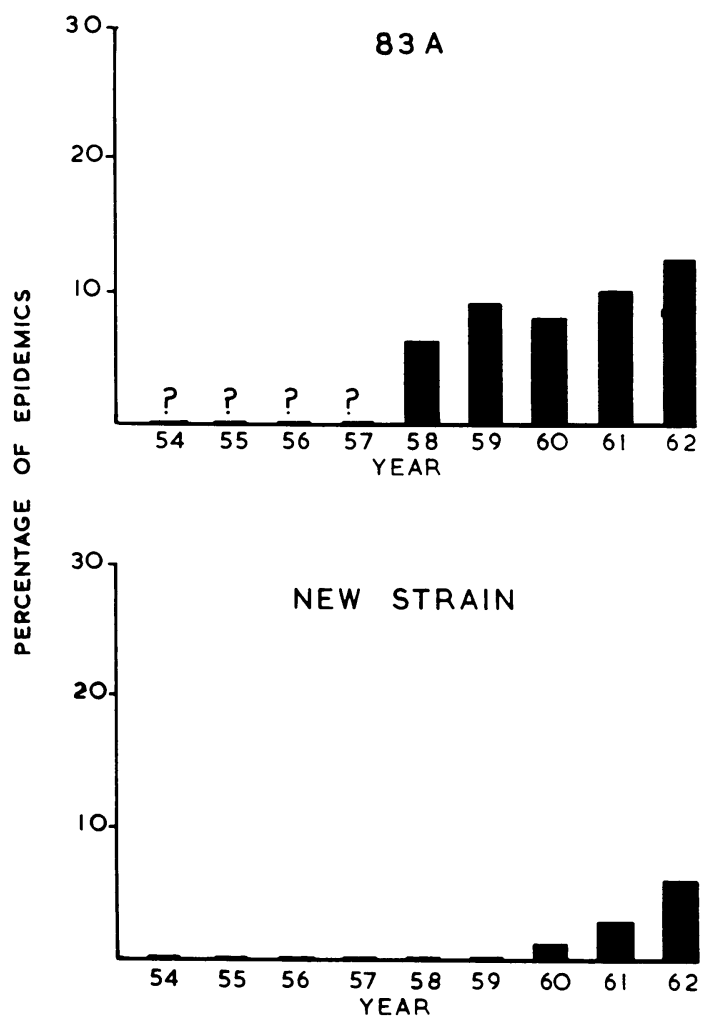

FIG. 2

since been identified in collections of cultures received from many foreign countries, but do not seem to have been observed in the United States and Canada.

A NEW UNTYPABLE STRAIN OF STAPHYLOCOCCUS AUREUS

Early in 1962 it became apparent that untypable organisms with a pattern of inhibition reactions with group III phages, but not lysed by phage $83 \mathrm{~A}$ (Fig. 1), were causing outbreaks of sepsis in England. Examination of our records showed that no such incidents were represented among the cultures sent to us in 1958 and 1959, that is to say, all the staphylococci with the characteristic inhibition pattern which gave rise to 'epidemics' were lysed by phage 83A. In 1960, however, we observed three such epidemics, and in the two subsequent years the numbers increased both absolutely and relatively (Fig. 2). Several accounts of local epidemics due to this organism in Great Britain have appeared. Temple and Blackburn (1963) described its prevalence in six hospitals in Glasgow; and soon afterwards Jacobs, Willis, Ludlam, and Goodburn (1963) in Leeds and 
Turner (1963) in Liverpool reported similar experiences. As long ago as 1960, similar untypable organisms recognizable by their inhibition reactions with group III phages were present endemically in hospitals in Cincinnati and other towns in the United States (Thomas, Hill, Culbertson, and Altemeier, 1960), and Comtois and Bynoe (1963) noted their appearances in Canada in 1961.

Like 83A strains, these new untypable organisms caused surgical sepsis and generalized infection in hospital patients, and were nearly always resistant to penicillin and tetracycline and to mercury salts.

\section{LYSIS OF THE NEW UNTYPABLE ORGANISM BY EXPERI- MENTAL PHAGES}

Although the new untypable organisms can usually be recognized easily by the presence of multiple inhibition reactions with group III phages, it would be an advantage if we had a phage to which they were all susceptible, in the same way as $83 \mathrm{~A}$ was found to lyse strains of a similar inhibition pattern in 1958. A number of experimental phages, all of which lysed some cultures of the new staphylococcus, have been examined, but none is entirely satisfactory. Their origin was as follows. Phage D was isolated by Wallmark and Finland (1961) in Boston, Mass., and found to lyse $3.2 \%$ of otherwise untypable strains there. Phage 77Ad was obtained in our laboratory from one of the scanty plaques appearing in the area of inhibition when the typing phage 77 was applied to one of the new untypable organisms
(9633). After purification, it was propagated on this same staphylococcus. Phage 12100 was supplied by Dr. Betty Clarke of the Royal Free Hospital, London. It was obtained from a lysogenic phage group III strain and propagated on an untypable staphylococcus (12088). Phage UC 18 (Thomas et al., 1960) was isolated in Cincinnatti, Ohio, and used to characterize a locally prevalent strain of untypable staphylococcus (propagating strain PS UC 18). Phage B5, from Dr. I. A. Porter of the University of Newcastle upon Tyne, had been used to recognize untypable strains in north east England (propagating strain PS B5).

Two hundred consecutively isolated untypable cultures which gave the characteristic inhibition pattern and 225 type $83 \mathrm{~A}$ staphylococci were tested with the first three of these phages (Table I). At R.T.D., phages D and 77Ad each lysed about three-quarters of the untypable cultures but phage 12100 lysed less than a fifth; even if all three were used as a pool $18 \%$ of the cultures showed no

\section{TABLE I}

REACTIONS OF NEW UNTYPABLE STAPHYLOCOCCI WITH EXPERIMENTAL PHAGES AT THEIR ROUTINE TEST DILUTIONS

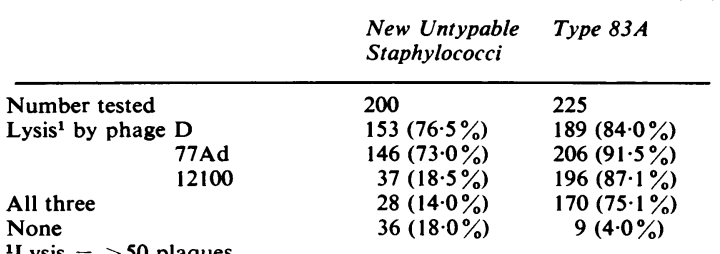

TABLE I I

PATTERNS OF SUSCEPTIBILITY OF REPRESENTATIVE UNTYPABlE CULTURES TO FIVE EXPERIMENTAL PHAGES Sources of Cultures ${ }^{1}$

\begin{tabular}{|c|c|c|c|c|}
\hline \multicolumn{5}{|l|}{ Phages } \\
\hline$D$ & $77 A d$ & 12100 & UC 18 & B 5 \\
\hline++ & ++ & $(++)$ & ++ & ++ \\
\hline$(++)$ & ++ & $(++)$ & ++ & ++ \\
\hline++ & ++ & ++ & - & ++ \\
\hline- & ++ & $(++)$ & ++ & ++ \\
\hline$(++)$ & ++ & ++ & - & ++ \\
\hline++ & ++ & $(++)$ & - & ++ \\
\hline++ & ++ & - & - & ++ \\
\hline++ & - & ++ & - & ++ \\
\hline- & ++ & - & ++ & ++ \\
\hline- & ++ & $(++)$ & - & ++ \\
\hline - & ++ & ++ & - & - \\
\hline- & - & ++ & - & ++ \\
\hline- & ++ & - & - & ++ \\
\hline$(++)$ & - & - & - & ++ \\
\hline- & - & ++ & - & $(++)$ \\
\hline- & $(++)$ & - & $(++)$ & - \\
\hline- & $(++)$ & - & - & $(++)$ \\
\hline 二 & $\begin{array}{c}(++) \\
-\end{array}$ & $\overline{-}$ & 二 & 二 \\
\hline
\end{tabular}

Richmond, Belfast 1, Papworth, Cincinnati 1, Ottawa

Glasgow 1, Cincinnati 2, Aylesbury 1, Liverpool

Kingston

Glasgow 2

London 1

Chertsey, London 2, London 3, London 4, Fife, Northampton

Aylesbury 2

London 5

Dumfries

Leeds 1

Bergen

Leeds 2

Birmingham

Leeds 3

Lima

London 6

Basle

Watford

London 7, Belfast 2, Glasgow 3, Geneva
'Thirty-four distinct strains from 25 hospitals. (Two organisms from the same hospital were considered distinct if their patterns of susceptibility differed by one or more strong reactions.) All gave multiple inhibition reactions with group III phages.

Strong reactions $++=>50$ plaques at R.T.D. $(++)=>50$ plaques at R.T.D. $\times 1,000 \quad$ Weak reactions Not recorded 
reaction. At R.T.D. $\times 1,000$, over half of the cultures were lysed by phage 12100 , but a small proportion gave no reaction with any of the phages. In general, 83A strains were more susceptible to the experimental phages than were the untypable strains.

When all five of the experimental phages were applied to a small collection of untypable strains from a variety of different geographical areas, many different pattern reactions were observed (Table II). Despite their superficial similarities, therefore, the new untypable organisms showed unexpected heterogeneity in their susceptibility to lysis by these phages.

\section{RELATION OF THE NEW UNTYPABLE STAPHYLOCOCCUS} TO TYPE 83A

The striking similarities between $83 \mathrm{~A}$ strains and the new untypable staphylococci suggested that they might be related, and that one might have arisen from the other by the loss or gain of a carried phage. The lysogenic state of the new untypable organism was therefore investigated.

ISOLATION OF BLOCKING PHAGES FROM UNTYPABLE STAPHYLOCOCCI Six cultures, including representatives of several different pattern reactions with the experimental phages, were selected. Washed suspensions of six-hour broth cultures were induced with ultra-violet light (Lwoff, Siminovitch, and Kjeldgaard, 1950), transferred to fresh broth and incubated for four hours. They were filtered through Oxoid membranes and spotted on to lawns of Staph. aureus 1116, a typical 83A organism. All six new untypable strains carried a phage or phages which lysed strain 1116. Each of the phages was purified by three single-plaque isolations and propagated on strain 1116 by the soft agar layer method of Swanstrom and Adams (1951).

The phages so obtained were designated by the number of the cultures from which they had been derived, with the prime sign (Anderson and Felix 1953): if more than one phage was obtained from a single culture, the subscript 1 or 2 was placed after the number. Thus $14368_{1}{ }^{\prime}$ and $14368_{2}{ }^{\prime}$ were two phages carried by culture 14368 .

Attempts were made to lysogenize the typical 83A culture (1116) with the phages obtained from the untypable staphylococci. High-titre preparations of the phages were spotted on to lawns of 1116 , and the plates were incubated overnight at $30^{\circ} \mathrm{C}$. The secondary growth in the areas of lysis was subcultured on to nutrient agar. Several of the resultant colonies were tested for lysogenicity by showing that they carried the lysogenizing phage and were resistant to it. All lysogenized cultures were typed with the routine set of phages. They are designated in the usual way; for example, $1116\left(1126^{\prime}\right)$ is staphylococcus 1116 carrying a phage from 1126 . At least one phage from each of the six untypableorganisms blocked the reaction of the $83 \mathrm{~A}$ culture with phage $83 \mathrm{~A}$, thus altering its typing pattern to that of a 'new untypable' staphylococcus.

One of the untypable organisms (1108) carried, in $\stackrel{\mathbb{2}}{\Omega}$ addition to the blocking phage, another phage which $\infty$ lysed 1116 but did not affect its reaction with $\vec{\circ}$

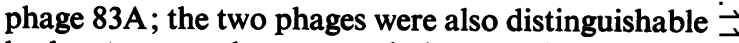
by host range, plaque morphology, and serological $\omega$ reaction. Strain 14368 carried two phages which blocked the reaction with phage $83 \mathrm{~A}$ on lyso-? genization, but differed from each other in host $\vec{v}$ range and serological group; strain 1116 lysogenized with either phage remained sensitive to the other. The six untypable strains carried seven blocking phages, four of which were distinguishable serologically, or by their lytic activity on the lysogenic derivatives of strain 1116. In all, five belonged to serological group $B$, one to group $A$, and one to group $\mathrm{F}$; phage $83 \mathrm{~A}$ itself belongs to serological group $B$.

ISOLATION OF NON-LYSOGENIC VARIANTS FROM UNTYPABLE STAPHYLOCOCCI If, indeed, the resistance of the untypable strains to phage $83 \mathrm{~A}$ is due to the carriage of a blocking phage, any non-lysogenic mutants present in the cultures should resemble 83A staphylococci in typing pattern.

Six-hour broth cultures of an untypable strain were flooded on to batches of nutrient agar plates containing $M / 50$ sodium citrate. They were exposed to ultra-violet light for a length of time sufficient to leave between 100 and 200 surviving colonies per plate after overnight incubation at $37^{\circ} \mathrm{C}$. These colonies were irradiated for a further 10 seconds immediately before replication by the method of Lederberg and Lederberg (1952).

Strains (such as 1126) shown to carry only one phage to which 1116 was sensitive were replicated to agar plates previously flooded with a broth culture of this organism. The plates were then incubated overnight at $30^{\circ} \mathrm{C}$., and colonies not surrounded by a halo of lysis were identified on the master plate, and tested for absence of carried phage and for sensitivity to phage 83A and the experimental phages.

Strain 1108, which was known to be carrying more than one phage which lysed strain 1116, was replicated to agar plates spread with $0.1 \mathrm{ml}$. of a high-titre preparation of phage 83A. After overnight incubation at $30^{\circ} \mathrm{C}$., the plates were compared with the master plate. It was assumed that any cocci failing to produce a colony on the replica plate had been lysed by phage 83A. The corresponding 
colonies on the master plates were therefore tested for lysogenicity and typing pattern.

Between 4,000 and 10,000 colonies of five different untypable cultures were screened for non-lysogenic variants. Only one such colony was obtained from one culture (1108), but the other four each yielded many, of which between three and five were selected for further examination. All the non-lysogenic variants from four of the five cultures were fully sensitive to phage $83 \mathrm{~A}$, and were thus indistinguishable from typical 83A strains. From the one exceptional culture (14364), three colonies were isolated which had lost the phage indicated by strain 1116 and was fully sensitive to phage $14364^{\prime}$, but only one of them was sensitive to phage 83A. This is being investigated further.

EFFECT OF LYSOGENIZATION ON SUSCEPTIBILITY TO EXPERIMENTAL PHAGES There is thus good evidence that many of the 'new untypables' could have arisen from type 83A strains by acquisition of a phage which blocks the reaction with phage 83A. The great variety of patterns of lysis of the new strains with the experimental phages, however, still had to be explained.

All the experimental work so far described was carried out with one 83A strain (1116), and the experimentally produced untypable strains were the result of lysogenization of it with blocking phages obtained from untypable strains from several different hospitals. When these lysogenic derivatives were tested with the experimental phages, however, their patterns of lysis were rather restricted; only $75 \%$ of the new untypable organisms isolated from routine material had patterns similar to those given by the lysogenic derivatives of strain 1116 .

There were several possible explanations of this: the 83A strains might not be as homogeneous in their characters as the results of typing with the basic set of phages suggested; they might carry other phages which influence susceptibility to the typing phages; additional, so far undetected, blocking phages might exist; and, possibly, some of the new untypable organisms might have arisen from the lysogenization of organisms that were not members of type 83A.

The first of these possibilities was examined. Although most 83A strains were lysed by all three of the experimental phages, $\mathrm{D}, 77 \mathrm{Ad}$, and 12100 , a minority were not (see Table I). Three further 83A cultures, two of which differed from 1116 in the pattern of their susceptibility to these three phages, were tested for their sensitivity to each of the seven blocking phages. Lysogenized cultures were obtained from all four organisms with each of the phages to which they were sensitive. The results are shown in Table III. Two new patterns of susceptibility to the

\section{TABLE III}

EFFECT OF LYSOGENIZATION WITH BLOCKING PHAGES ON THE SUSCEPTIBILITY OF CERTAIN 83A CULTURES TO THREE EXPERIMENTAL PHAGES

Strain

Typing Pattern

Serological Group

$83 A$
Pattern No. Percentage Routine Strains with Similar Pattern

\section{6}

$1116\left(1108_{1}^{\prime}\right)$

$1116\left(1126^{\prime}\right)$

$1116\left(14361^{\prime}\right)$

$1116\left(14368_{1}{ }^{\prime}\right)$

$1116\left(14368_{z}^{\prime}\right)$

$1116\left(9514^{\prime}\right)$

1116 (13564)

1124

$1124\left(1108_{1}^{\prime}\right)$

$1124\left(1126^{\prime}\right)$

$1124\left(14361^{\prime}\right)$

$1124\left(14368_{1}^{\prime}\right)$

$1124\left(14368_{2}^{\prime}\right)$

1124 (9514')

1124 (13564')

443

$443\left(1108_{1}{ }^{\prime}\right)$

$443\left(14368_{8}^{\prime}\right)$

36

36 (1126')

$36\left(14361^{\prime}\right)$

$36\left(14368_{1}^{\prime}\right)$

$36\left(14368_{2}^{\prime}\right)$

Total

83A
Untypable
Untypable
Untypable
Untypable
Untypable
Untypable
Untypable
83A
Untypable
Untypable
Untypable
Untypable
Untypable
Untypable
Untypable
83A
Untypable
Untypable
83A
Untypable
Untypable
Untypable
Untypable

${ }^{1}$ Titre of phages not less than R.T.D. $\times 1,000$.

$+=$ lysis

$-=$ no lysis 
experimental phages resulted, so that $95 \%$ of the patterns seen among new untypable organisms isolated from routine material were also found among the lysogenic derivatives of two $83 \mathrm{~A}$ cultures.

Further inspection of Table III shows that the pattern of susceptibility of the artificially produced untypable strain is determined not only by the pattern of the 83A strain from which it is derived, but also by the identity of the lysogenizing phage; and that the lysogenization of different cultures with the same phage sometimes blocks a particular reaction and sometimes does not. For example, cultures 1124 and 1116 are both sensitive to phage 77Ad, but $1124\left(14361^{\prime}\right)$ is resistant and $1116\left(14361^{\prime}\right)$ is sensitive to this phage. Even when two $83 \mathrm{~A}$ cultures had the same pattern of susceptibility to the experimental phages, e.g., 1116 and 36, lysogenization with the same phage, e.g., 14361', had different results.

Three of the blocking phages $\left(1126^{\prime}, 14361^{\prime}\right.$, and $9514^{\prime}$ ) belonged to the same serological group. When used to lysogenize strain 1116 they caused the same changes in its pattern of susceptibility to the experimental phages. The results of lysogenization of other cultures with the same phages make it clear, however, that they are not identical. Thus 1124 $\left(1126^{\prime}\right)$ is sensitive to phage 77Ad but $1124\left(14361^{\prime}\right)$ is not; and phage $9514^{\prime}$ is different from $1126^{\prime}$ and $14361^{\prime}$ in that it fails to lyse strains 36 and 443.

Whether carried phages other than those which block the reactions with phage $83 \mathrm{~A}$ also influence the typing pattern of naturally occurring strains is uncertain. One phage $\left(1108_{2}{ }^{\prime}\right)$ isolated from an untypable organism together with a blocking phage also lysed 83A strains. There was no evidence that lysogenization of three $83 \mathrm{~A}$ strains with this phage resulted in any alteration of susceptibility of the experimental phages.

\section{DISCUSSION}

The recent appearance in British hospitals of a number of somewhat similar untypable staphylococci afforded a further opportunity to investigate the part played by lysogenicity in determining the phage-typing patterns of Staph. aureus.

In 1954 an important new hospital strain of Staph. aureus, which was untypable by the phages then in use, was recognized first in Australia (Isbister, Durie, Rountree, and Freeman, 1954) and soon afterwards in many other places (Rountree and Beard, 1958). The phage 80, and later also phage 81, was introduced to characterize it (Rountree and Freeman, 1955; Bynoe, Elder, and Comtois, 1956). Figure 3 illustrates our experience with this organism in Great Britain; it shows the percentage

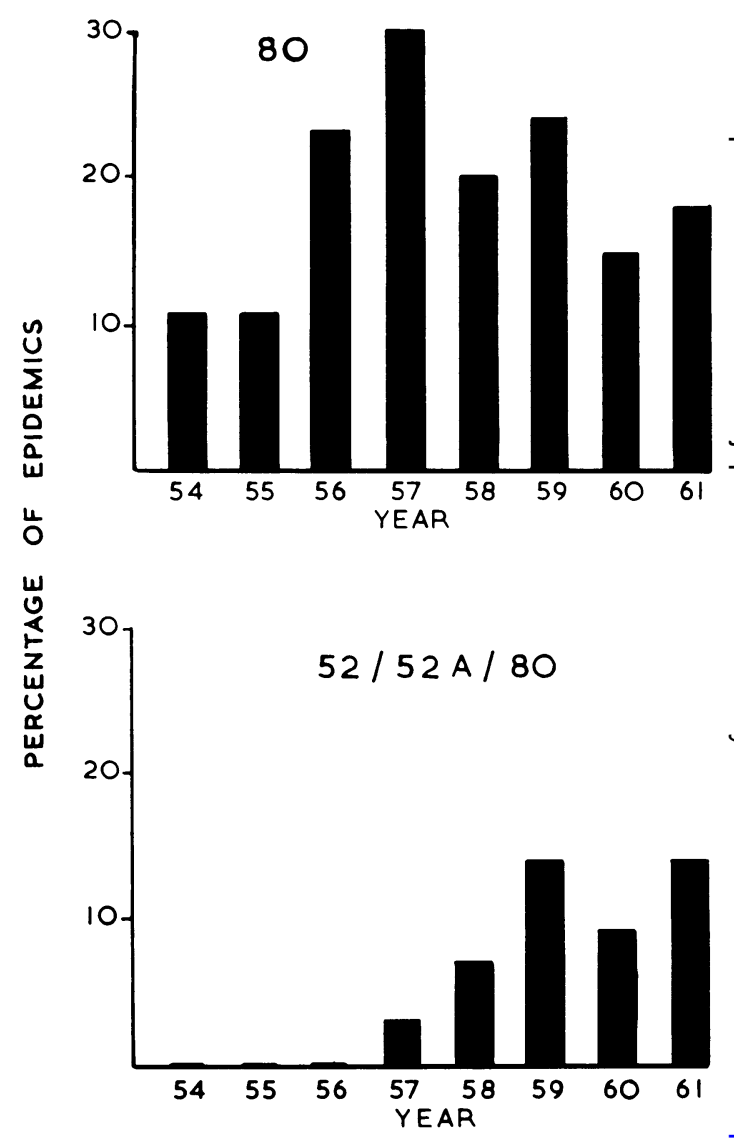

FIG. 3. Percentage of epidemics of hospital sepsis that were due to strains of Staph. aureus with the phage typing patterns (a) 80 (or 80/81) and (b) 52/52A/80 (or 52/52A $80 / 81$ ). Phage 81 was used only in 1961.

of 'epidemics' (incidents consisting of three or more related septic lesions) due to 80 or $80 / 81$ strains represented among the cultures received for typing at the Staphylococcus Reference Laboratory, Colindale, mainly from hospitals in London and the south of England. Type 80 strains were already prevalent when these records were begun in 1954, and increased in frequency during the next few years. From 1957 onwards, however, similar incidents were observed associated with organisms lysed by phages $52,52 \mathrm{~A}$, and 80 (or $52,52 \mathrm{~A}, 80$, and 81 , appearing at first in hospitals in which type 80 infections were already endemic (Asheshov and Rippon, 1959). It was soon shown (Rountree, 1959; Asheshov and Rippon, 1959) that 80/81 strains could be converted to $52 / 52 \mathrm{~A} / 80 / 81$ strains by lysogenization with one of a number of phages 
carried by related staphylococci. The hospital $52 / 52 \mathrm{~A} / 80 / 81$ strains were therefore seen as $80 / 81$ strains with a changed typing pattern due to lysogenization, and the idea arose of a 52, 52A, 80, 81 complex, a group of strains lysed by one or more of these phages and no others, and interconvertible by the loss or gain of a carried phage (Rountree and Asheshov, 1961).

The parallelism between these events and the changes reported in this paper may be closer than might appear at first sight. The acquisition of a carried phage by a staphylococcus usually results in a loss of susceptibility to lysis by other phages (Lowbury and Hood, 1953). The broadening of typing pattern from $80 / 81$ to $52 / 52 \mathrm{~A} / 80 / 81$ by lysogenization was unexplained until Rountree and Asheshov (1961) showed that the insusceptibility of $80 / 81$ strains to phages 52 and $52 \mathrm{~A}$ was due to the carriage of a defective phage which could be replaced by certain phages on lysogenization with them. In this case, therefore, the consequence of lysogenization was effectively the loss of the blocking phage. Sompolinsky (1963), in Israel, described another change in the $52,52 \mathrm{~A}, 80,81$, complex which was even more like recent events in Great Britain. He noticed the appearance of an untypable strain in hospitals in which type 80/81 staphylococci were endemic, and was able to make similar untypable organisms by lysogenization of type $80 / 81$ staphylococci with a blocking phage from the untypable strain.

The investigations reported here suggest that most of the new untypable staphylococci that have appeared recently in this country arose from members of type $83 \mathrm{~A}$ by the acquisition of a blocking phage. Six out of six untypable organisms carried a blocking phage for the $83 \mathrm{~A}$ reaction, and non-lysogenic variants of four out of four organisms were lysed by phage $83 \mathrm{~A}$.

The possibility remains, however, that similar organisms might be produced by the lysogenization of other staphylococci. The fact that untypable organisms very like those recently seen in Great Britain have also been prevalent in the United States and Canada from 1959 onwards, but that members of type 83A had not been seen there (Comtois and Bynoe, 1963), reinforces this suggestion. Further investigations are in progress to determine the precursors of the untypable organisms isolated in other countries.

Our enquiries also revealed an unexpected heterogeneity both among the new untypable organisms and in type $83 \mathrm{~A}$ itself. This was rather surprising, because several authors had observed that the new untypable organisms often had several unusual cultural characters, including lemon-yellow pigmentation on glycerol monoacetate agar, the production of $\beta$ lysin and absence of fibrinolysin (Turner and Willis, 1962; Willis, Jacobs, and Goodburn, 1963), and neomycin resistance (Robertson, 1963). The distribution of these and other characters will form the subject of a further communication.

It is interesting to speculate on the part played by the choice of typing phages on the epidemiological picture that we obtain. If phage $D$ had been introduced into our basic typing set in 1958, instead of phage $83 \mathrm{~A}$, the recent events would probably have passed unnoticed, since the new untypable organisms would have been indistinguishable from type 83A by the phage typing method. The somewhat similar organism which became common in North America from 1959 onwards would also have been placed in the same type. Phage-typing patterns have in the past directed attention to undoubted differences in the epidemiological behaviour of particular staphylococcal strains, but it would be unwise to assume that organisms with the same susceptibility to typing phages necessarily resemble each other in every other way.

We can no longer speak of staphylococcus phage types as if they are distinct parcels of organisms, unchanging in all their characters. Fortunately, this detracts very little from the practical value of phage typing in short-term epidemiological investigations. What should interest the clinician is not the so-called type of the organism, but the source of the infection; and the answer to the classical question, 'Could A have infected B?', can still be given with some confidence.

In long-term studies, however, it is a different matter. We now realize that the enormous numbers of different staphylococci in the environment, and their state of multiple lysogenicity, affords them such opportunities for prophage exchange as to cause confusion among those seeking to establish a static system for classifying staphylococci. This is not to say that all our fixed points are likely to disappear. We are tending to think of Staph. aureus as a series of complexes of related strains within which evolutionary change is taking place. Phage typing will continue to be of interest if we understand the limits within which changes in the lysogenic state can influence other characters of the organism.

\section{REFERENCES}

Anderson, E. S., and Felix, A. (1953). J. gen. Microbiol., 9, 65. Anderson, E. S., and Felix, A. (1953). J. gen. Microbiol.,

Asheshov, E. H., and Rippon, J. E. (1959). J. gen. Microbiol., 20, 634. Blair, J. E., and Williams, R. E. O. (1961). Bull. Wld Hlth Org., 24, 771. Bynoe, E. T., Elder, R. H., and Comtois, R. D. (1956). Canad. J. Microbiol., 2, 346.

Comtois, R. D., and Bynoe, E. T. (1963). Canad. J. publ. Hlth, 54, 537. 
Isbister, C., Durie, E. B., Rountree, P. M., and Freeman, B. M. (1954). Med. J. Aust., 2, 897.

Jacobs, S. I., Willis, A. T., Ludlam, G. B., and Goodburn, G. M. (1963). Lancet, 1, 972 .

Lederberg, J., and Lederberg, E. M. (1952). J. Bact., 63, 399.

Lowbury, E. J. L., and Hood, A. M. (1953). J. gen. Microbiol., 9, 524

Lwoff, A., Siminovitch, L., and Kjeldgaard, N. (1950). Ann. Inst. Pasteur, 79, 815

Parker, M. T. (1962). J. appl. Bact., 25, 389.

Report. (1963). Int. Bull. bact. Nomencl., 13, 119.

Robertson, J. J. (1963). Lancet, 2, 333.

Rountree, P. M. (1959). J. gen. Microbiol., 20, 620.

-, and Asheshov, E. H. (1961). Ibid., 26, 111.

_-, and Beard, M. A. (1958). Med. J. Aust., 2, 789.
- and Freeman, B. M. (1955). Ibid., 2, 157.

Swanstrom, D. (1963). Amer. M. H. (1951). Proc. Soc exp. Biol. (N.Y.), 78, 372 .

Temple, N. E. I., and Blackburn, E. A. (1963). Lancet, 1, 581.

Thomas, D. C. Hill, E. O., Culbertson, W. R., and Altemeier, W. A. (1960). Surg. Forum, 10, 334.

Turner, G. C. (1963). Lancet, 1, 1156.

- and Willis, A. T. (1962). J. Path. Bact., 84, 349.

Wallmark, G., and Finland, M. (1961). Proc. Soc. exp. Biol. (N.Y.), 106, 73.

Williams, R. E. O., and Jevons, M. P. (1961). Zbl. Bakt., 1. Abt. Orig., $181,349$.

Willis, A. T., Jacobs, S. I., and Goodburn, G. M. (1963). Lancet, 2, 67. 